\title{
Prospective Head Motion Compensation for MRI by Updating the Gradients and Radio Frequency During Data Acquisition
}

\author{
Christian Dold ${ }^{1}$, Maxim Zaitsev ${ }^{2}$, Oliver Speck ${ }^{2}$, Evelyn A. Firle ${ }^{1}$, \\ Jürgen Hennig ${ }^{2}$, and Georgios Sakas ${ }^{1}$ \\ 1 Fraunhofer Institute for Computer Graphics, \\ Dept. Cognitive Computing \& Medical Imaging, Darmstadt, Germany \\ 2 University Hospital Freiburg, Dept. of Diagnostic Radiology, \\ Medical Physics, Freiburg, Germany \\ Christian.Dold@igd.fraunhofer.de
}

\begin{abstract}
Subject motion appears to be a limiting factor in numerous magnetic resonance imaging (MRI) applications. For head imaging the subject's ability to maintain the same head position for a considerable period of time places restrictions on the total acquisition time. For healthy individuals this time typically does not exceed 10 minutes and may be considerably reduced in case of pathology. In particular, head tremor, which often accompanies stroke, may render certain high-resolution 2D and 3D techniques inapplicable. Several navigator techniques have been proposed to circumvent the subject motion problem. The most suitable for head imaging appears to be the orbital or spherical navigator methods. Navigators, however, not only lengthen the measurement because of the time required for acquisition of the position information, but also require additional excitation radio frequency $(\mathrm{RF})$ pulses to be incorporated into the sequence timing, which disturbs the steady state. Here we demonstrate the possibility of interfacing the MR scanner with an external optical motion tracking system, capable of determining the object's position with sub-millimeter accuracy and an update rate of $60 \mathrm{~Hz}$. The movement information on the object position (head) is used to compensate the motion in real time. This is done by updating the field of view (FOV) by recalculating the gradients and the RF-parameter of the MRI tomograph during the acquisition of $\mathrm{k}$-space data based on the tracking data. Results of rotation phantom, in vivo experiments and the implementation in two different MRI sequences are presented.
\end{abstract}

\section{Introduction}

Patient motion remains a significant problem in many MRI applications, including fMRI 1,2, cardiac- and abdominal imaging as well as conventional acquisitions. Many techniques are available to reduce or to compensate for bulk motion effects, such as physiological gating, phase-encode reordering, fiducial markers [3, special algorithms [4, fast acquisitions, image volume registration, or alternative data acquisition strategies such as projection reconstruction, spiral and PROPELLER [5, 6. Navigator echoes are used to measure motion with one or 


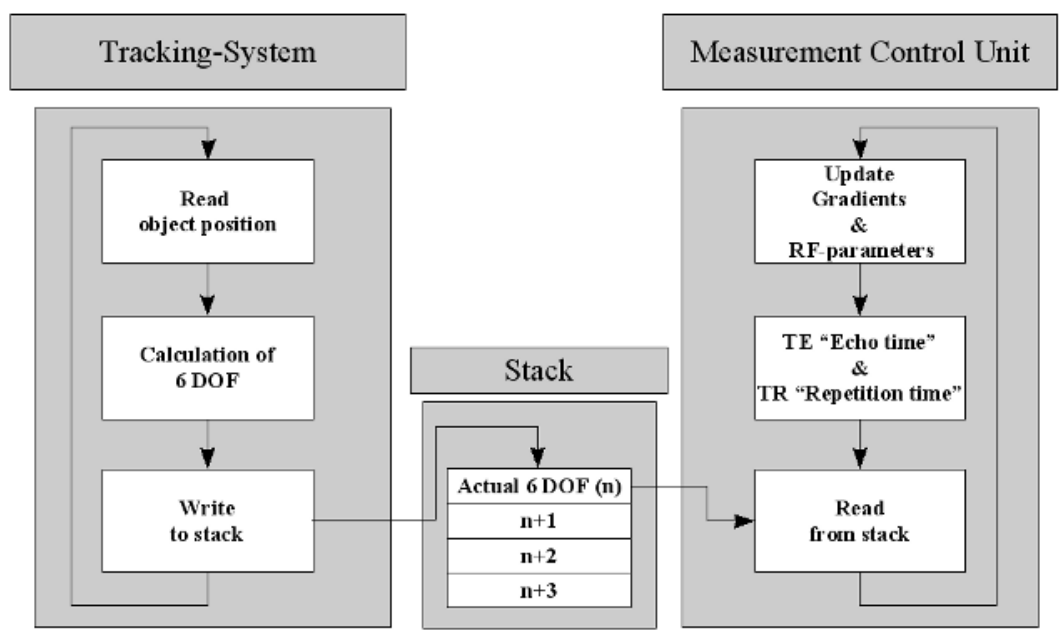

Fig. 1. The setup of the data handling to update the field of view depending on the subject motion

more degrees of freedom [7]. The motion is then compensated for either retrospectively or prospectively. An orbital navigator (ONAV) echo captures data in a circle in some plane of k-space, centered at the origin [8-10]. This data can be used to detect rotational and translational motion in this plane, and to correct for this motion. However, multiple orthogonal ONAVs are required for general 3D motion determination, and the accuracy of a given ONAV is adversely affected by motion out of its plane. Methods capable of correcting for head motion in all six degrees of freedom have been proposed for human Positron Emission Tomography (PET) brain imaging [11. These methods rely on the accurate measurement of head motion in relation to the reconstruction coordinate frame. Implementing a similar technique in MRI presents additional challenges. Foremost the tracking system and the MRI system have to be compatible. High magnetic fields $\geq 1.5$ Tesla in MRI systems require that the tracking camera system be positioned at a sufficient distance from the MRI system to ensure proper function and safety. Aditionally, efforts need to be taken to ensure radiofrequency screening on the tracking hardware. Functional MRI also proves challenging because of the high spatial accuracy (root mean square $(\mathrm{RMS})<0,3 \mathrm{~mm}$ ) required by the complete measurement chain with a small latency time of the tracking system. A precise relationship between the spatially varying magnetic field gradients and the spatial tracking information is necessary to compensate for motion artifacts. Our initial trials using an external tracking system to compensate for movement artifacts in MRI are published in [12].

\section{Material and Methods}

The technique was implemented on a Siemens Magnetom Trio 3T whole-body system (Siemens Medical Systems $\mathrm{GmbH}$ ) at the University Hospital Freiburg. 


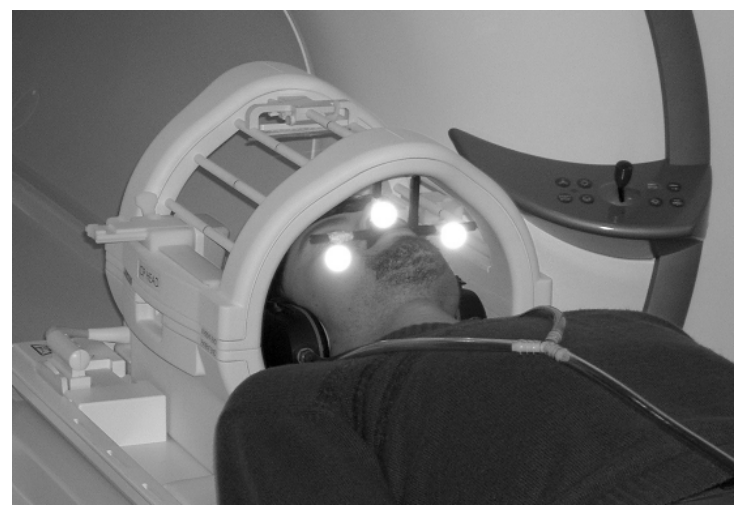

Fig. 2. A volunteer with a mouthpiece inside the head-coil but outside of the scanner

The cameras of the optical motion tracking system (ARTrack1) together with an own developed software has been used to create a stereoscopic reconstruction of rigid bodies from gray scale images. The tracking system was capable of reporting positions and orientations of rigid targets fitted with "passive" retro reflective markers in six degrees of freedom (6DOF) using two progressive scan cameras synchronized by a sync card [13. The passive targets consisted of at least four coplanar retro reflective markers. All markers were filled with doped water to be detectable by both MR and the optical tracking system. The cameras were equipped with infrared flashes to illuminate the scene with infrared light. Several targets could be tracked simultaneously at a sampling rate of up to $60 \mathrm{~Hz}$, with a quoted positional accuracy less than $0.1 \mathrm{~mm}$ (RMS).

Our goal was to prospectively correct artifacts by tracking the head motion by means of the camera immediately before performing the acquisition and then use the motion data in order to internally re-position the coordinate system of the scanner on-line for performing the next scan. A small latency between measuring, scanner coordinates updating and scan performance is essential for the success of the method. Communication with the measurement control unit (MCU) of the MRI instrument took place over a TCP/IP connection. During the calibration step (performed once) the point of origin of the tracking system was transformed to the physical center of the gradient system and the corresponding coordinate transformation matrix has been calculated to overlap both coordinate systems. The information on the target position and orientation was used by the MRI system to update the position of the imaging volume in real time by means of respective transformations of gradients and radiofrequency shifts. For this purpose the scanner software is changed as well. As a result, scanning of sub-sequent planes or lines are aligned to the first acquisition and, thus, the target appears to be virtually immobilised and without motion artifacts.

Phantom scans as well as in vivo imaging experiments were performed with healthy volunteers. All experiments with human subjects were performed in ac- 
cordance with the guidance of the local institutional review board (IRB) regulations and the informed consents were obtained prior to measurements.

\subsection{Phantom Tests with Small and Large Rotations}

The rotation of the phantom was realized manually in the MRI. No rotation, small rotation up to 5 degree and large rotation up to 20 degree in each direction were done by motion correction (Mo-Co) enabled/ disabled as can be seen in the figure: 3. Every translation and rotation value (6DOF) is stored in a log file. The motion correction was performed prospectively by updating the MR-gradients $(\mathrm{x}, \mathrm{y}, \mathrm{z})$ and the RF-parameter based on the tracking information.

\subsection{Slice by Slice Correction for Echo Planar Imaging (EPI)}

The EPI sequence was modified to enable real-time slice-by-slice feedback from the MCU. The latter was communicating with the optical motion tracking system and generating the feedback information for the measurement. Imaging parameters were as follows: $\mathrm{FOV}=256 \mathrm{~mm}$, image matrix of $64^{2}, 32$ slices, $4 \mathrm{~mm}$ slice thickness, $1.2 \mathrm{~mm}$ slice gap, interleaved multi-slice acquisition, echo delay time (TE) was $16 \mathrm{~ms}$, repetition time (TR) was $1660 \mathrm{~ms}$. In order to track position of the subject's head a mouthpiece with 4 retro-reflective markers was used. Subjects were instructed to bite the mouthpiece tightly to make sure it

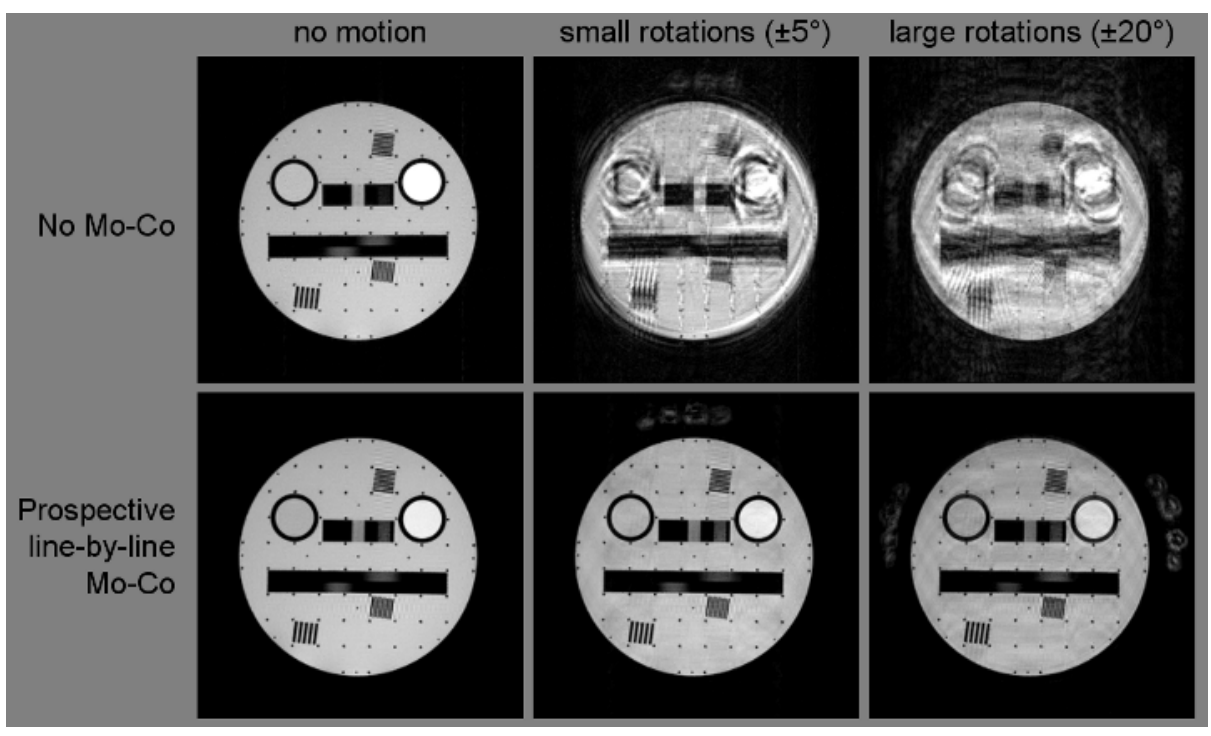

Fig. 3. 2D spin echo images in the top raw are shown with motion correction (Mo-Co) disabled and in the bottom raw the ones with Mo-Co enabled. Motion compensation was done in real-time by using the data delivered by the tracking system to update orientation and position of the imaging volume. 
remains in contact with the upper jaw in order to make the system of 4 markers and the scull a rigid body. No head fixation pads were used in order to enable exaggerated motion during the imaging experiments.

\subsection{The Line by line Correction for Turbo Spin Echo (TSE) Sequence}

The TSE sequence is a pulse sequence characterized by a series of rapidly applied $180^{\circ}$ rephasing pulses and multiple echoes which resulting in shorter scan times. The product TSE sequence was modified to enable real-time line-by-line motion correction. Imaging parameters were: $\mathrm{FOV}=224 \mathrm{~mm}$, image matrix of $256^{2}$, $\mathrm{TE}=12 \mathrm{~ms}, \mathrm{TR}=500 \mathrm{~ms}$, phase-encoding dimension was vertical from anterior to posterior $(\mathrm{AP})$.

\section{Result}

The positional accuracy of the tracking system with an FOV of $50 \mathrm{~cm}^{3}$ was better than $0.1 \mathrm{~mm}$ (RMS). The mean value of the measured reproducibility of the rotation and translation was 0.008 degree $/ 0.067 \mathrm{~mm}$. No artifacts were detected in the MR images originating from the possible interactions of the MR system and the tracking system. The latency time of the whole measurement chain and correction was $30 \mathrm{~ms}$. The prospective slice-by-slice motion correction avoids unrecoverable volume distortions. The remaining modulation may be attributed to EPI geometric distortions, which are known to be position-dependent (figure: 4).
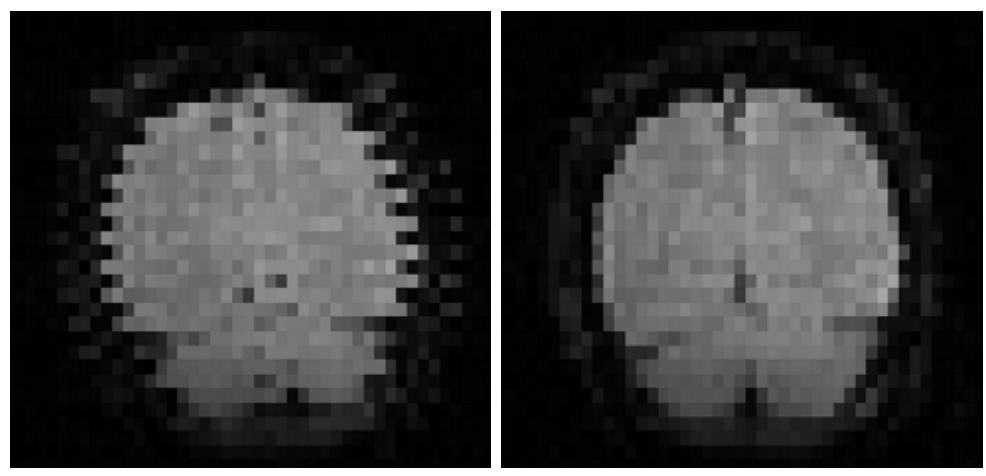

Fig. 4. Representative coronal images produced by re-slicing transversal EPI data from the interleaved multi-slice acquisition. Severe displacements between the neighboring slices are apparent when motion occurs during the acquisition of a single volume (left). Adjusting the position of each slice circumvents the intra-volume distortion problem (right). The remaining modulation and/or step-like structures at the surface of the brain are due to geometric distortions of EPI, which can change with the orientation of the head. The motion corresponds to a single continuous head rotation of yaw axis. 


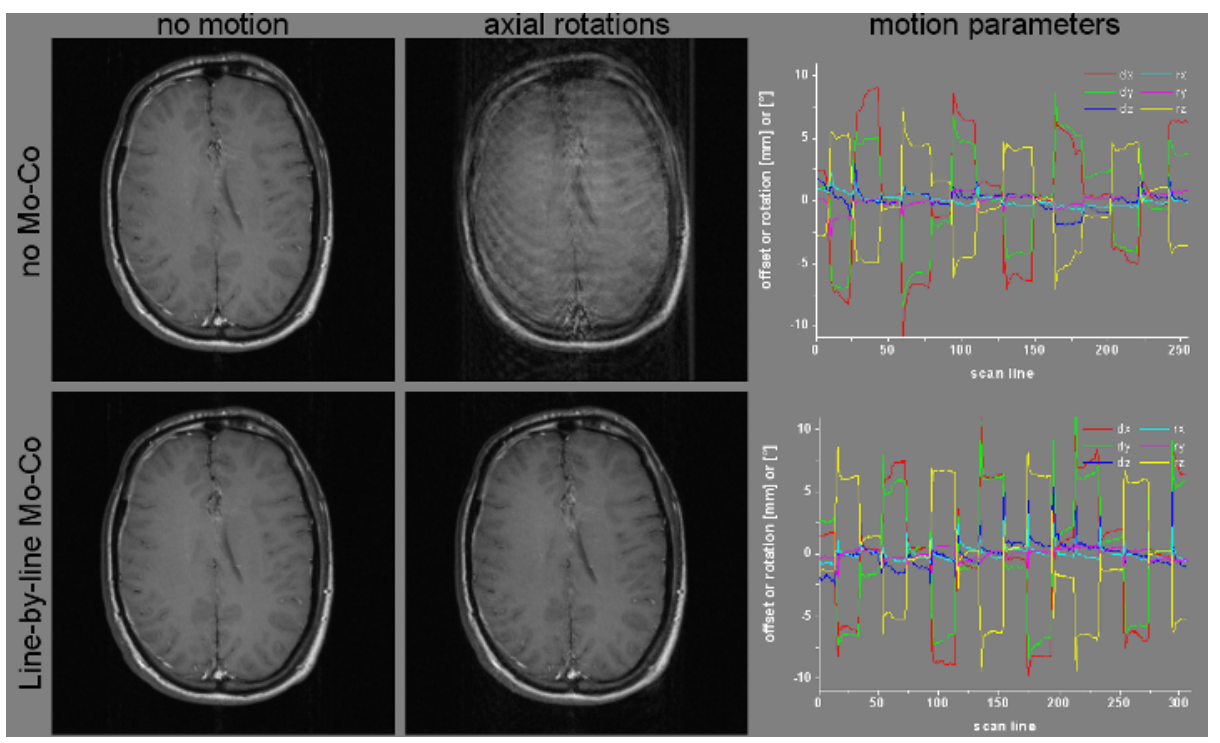

Fig. 5. Brain spin-echo images in the absence of subject motion, motion correction disabled (top left) and enabled (bottom left). Strong subject motion with Mo-Co disabled (top middle) and enabled (bottom middle). Corresponding motion patterns are presented (top/ bottom right). They are shown similar motion values to each other, but the number of scanned lines was in the corrected one higher because of the rejected $\mathrm{k}$-lines including to much motion. Significant reduction of artifacts by the motion correction is apparent. In fact the corrected image shows effectively the same quality as the reference one, while the uncorrected one is useless for medical purposes!

The result of the method demonstrate the advantages of optical motion tracking to correct for motion in echo-planar time series by not only enabling volumeto-volume correction without additional computational overhead, but also sliceby-slice correction without increasing the scanning time.

In figure: [5] a slice through the brain of a normal volunteer is presented, acquired in 4 measurements. In the first two experiments the volunteer was instructed not to move the head. The figure:5top left was acquired with the motion correction disabled and serves as a reference for the maximum achievable image quality. Figure 5 bottom left was acquired with motion correction enabled but still with unmoved patient in the gantry. Given the absence of subject motion, the experiment has been done in order to visualise the effects of "camera noise" or "jittering effects" visible as an increasing of image noise and slight artifacts in phase encoding dimension due to the uncertainty of the position determination generated by the noising of the optical motion tracking system. In our case these effects were not only negligible compared to the usual physiological motion of the head of living persons, but above this the acquired image shows even improved sharpness and contrast as compared to each other with the software IQM (Image Power Spectrum) [14 and 5 radiologists in an indepentent questioning. 
In the further two experiments the volunteer was instructed to move the head during the acquisition. In figure: 5 top middle the resulting image quality showing significant artifacts with the motion correction disabled. The figure: 5 bottom middle presents the image acquired with imaging coordinates updated for each $k$-space line. The corresponding motion patterns are presented in figure: 5 top right and top left. As can be seen, for comparable motion patterns, the motion artifacts are much reduced, when prospective motion correction is performed.

\section{Discussion}

We presented a motion correction method which is working in real-time only by updating the FOV of the MRI tomograph. The movement term, acquired through the tracking system, is transfered to the MRI tomograph and used for adapting the gradients and RF-parameter to compensate the complete motion. The results show the feasibility of using an external optical motion tracking system to correct for subject motion during the acquisition of a single image on a line by-line basis ( $k$-space line).

Even without any intended motion the image in figure: 5 bottom left is improved in comparison to the reference in figure: 5] top left. This signifies that the noise in the position data generated by the tracking system, introduces less artifacts than the minimal, physiologically unavoidable movement of the head during data acquisition. As a result, even such minimal movements can be compensated resulting in visible quality improvements also for co-operative patients. In case of moving subject, the corrected image acquired in the presence of motion (figure: 5 bottom middle) shows considerable quality improvement in comparison to the image acquired in the presence of comparable motion without correction (figure: [5 top middle). The latency time of the whole measurement chain was now reduced to approximately $30 \mathrm{~ms}$. Doing movement correction within echo- and repetition time to minimize phase effects appears to be possible. This further improvement needs a movement detection and a adjustment of the gradients and RF-parameter in less than $10 \mathrm{~ms}$. One of the limitation, correcting movement artifacts, is always the non linearity of the gradients in the magnetic field. As further limitation can be seen the small inaccuracy of the cross-calibration between the MRI system and the tracking device and the lag in the motion data can also contribute to the reduction of image quality.

Expected benefits of our technique include a significant reduction in imaging time since we perform prospective correction, no oversampling and we avoid repeating acquisitions corrupted by motion. Therefore, our technique helps to increase the clinical efficiency, the patient throughput and reduces measurement redundancy. In the near future the proposed method may be used to examine non-cooperative patients, e.g. the difficult and dangerous tranquillisation of children with sedatives is to become redundant. 


\section{Acknowledgement}

The research work is funded by the European Union IST (Information Society Technologies) program. The project is named MRI-MARCB.

\section{References}

1. Babak A. Ardekani et al.: A quantitative comparison of motion detection algorithms in fMRI. Magn. Reson. Imag. 19:959-963, 2001.

2. Lee CC et al.: Real-time adaptive motion correction in functional MRI. Magn. Reson. Med. 36:436-444, 1996.

3. Korin HW, Felmlee JP, Riederer SJ, Ehman RL: Spatial-frequency-tuned markers and adaptive correction for rotational motion. Magn. Reson. Med. 33:663-669, 1995.

4. Shankaranarayanan A et al.: Two-step navigatorless correction algorithm for radial k-space MRI acquisitions. Magn. Reson. Med. 45:277-288, 2001.

5. Pipe JG et al.: Motion correction with PROPELLER MRI: Application to head motion and free-breathing cardiac imaging. Magn. Reson. Med. 42:963-969, 1999.

6. Starty GE et al.: Single TrAjectory Radial (STAR) Imaging. Magn. Reson. Med. 51:445-451, 2004.

7. Thesen S et al.: Prospective acquisition correction for head motion with imagebased tracking for real-time MRI. Magn. Reson. Med. 44:457-465, 2000.

8. Lee CC et al.: A prospective approach to correct for inter-image head rotation in fMRI. Magn. Reson. Med. 39:234-243, 1998.

9. Fu ZW et al.: Orbital navigator echoes for motion measurements in magnetic resonance imaging. Magn. Reson. Med. 34:746-753, 1995.

10. Welch EB et al.: Spherical Navigator Echoes for Full 3D Rigid Body Motion Measurement in MRI. Magn. Res. Med. 47:32-41, 2002.

11. R. Fulton et al.: Correction for Head Movements in Positron Emission Tomography Using an Optical Motion-Tracking System. IEEE Trans. Nucl. Sci. 49:116-123, 2002 .

12. C. Dold et al.: The Compensation of Head Motion Artifacts using an Infrared Tracking System and a new Algorithm for fMRI. Medicine Meets Virtual Reality 12:75-81, 2004.

13. A. Wagner et al.: Quantitative analysis of factors affecting intraoperative precision and stability of optoelectronic and electromagnetic tracking systems. Medical Physics Vol. 29(5):905-912, 2002.

14. N.B. Nill and B.H.Bouzas et al.: Objective Image Quality Measure Derived from Digital Image Power Spectra. Optical Engineering 31:813-825, 1992. 